\title{
Original
}

\section{The Effectiveness of Simultaneous Combination Therapy Using S-1 and Nedaplatin with Radiation for Five Cases of Maxillary Cancer}

\author{
Toshikazu Shimane ${ }^{1)}$, Tomoaki Mori ${ }^{1)}$, Tomohiro ONO ${ }^{1)}$, \\ Tetsuya Monden $^{1)}$, Ayako Furuya ${ }^{1)}$, Takeyuki SAnBe ${ }^{1)}$ \\ and Harumi SuZAKI ${ }^{2)}$
}

\begin{abstract}
In our department, we provide treatment for malignant head and neck tumors with the goal of preserving organs and function. We report the treatment outcomes for five patients with maxillary cancer who underwent S-1 and nedaplatin treatment with concurrent radiation therapy (SN therapy) in our department from April 2005 to March 2009. The patients included one case classified as T2N0M0 and four cases classified as T4N0M0. All patients were male and were aged between 29 and 67 years with a mean age of 55.6 years. Among the five cases, four achieved cancer-free survival with preservation of all functions after undergoing treatment. In the remaining case, the tumor did not disappear but was locally controlled via superselective arterial injection and CyberKnife treatment; however, pulmonary metastasis occurred and the patient is surviving with cancer. SN therapy has allowed a reduction in the extent of surgery required and the preservation of organs and function. It will be necessary to study an increased number of cases in the future to determine the survival rate and the effectiveness of organ and function preservation in maxillary cancer patients after SN therapy.
\end{abstract}

Key words : maxillary cancer, SN therapy, concurrent chemoradiotherapy

\section{Introduction}

Tumors of the nasal cavity and paranasal sinus are rare among head and neck neoplasms. However, the tumor types in this region vary widely and early detection is difficult because of the anatomical location. There have been many cases in which the tumor has grown extensively before the appearance of any symptoms.

For maxillary cancer, various reports have addressed the effects of radiation therapy, chemotherapy with radiation therapy (including arterial injection), en bloc resection by surgery, and fractional excision. At present, there are wide interfacility variations in regard to the

\footnotetext{
1) Department of Otorhinolaryngology, Showa University, Fujigaoka Hospital, 1-30 Fujigaoka, Aoba-ku, Yokohama 227-8501, Japan.

2) Department of Otorhinolaryngology, Showa University School of Medicine.
} 


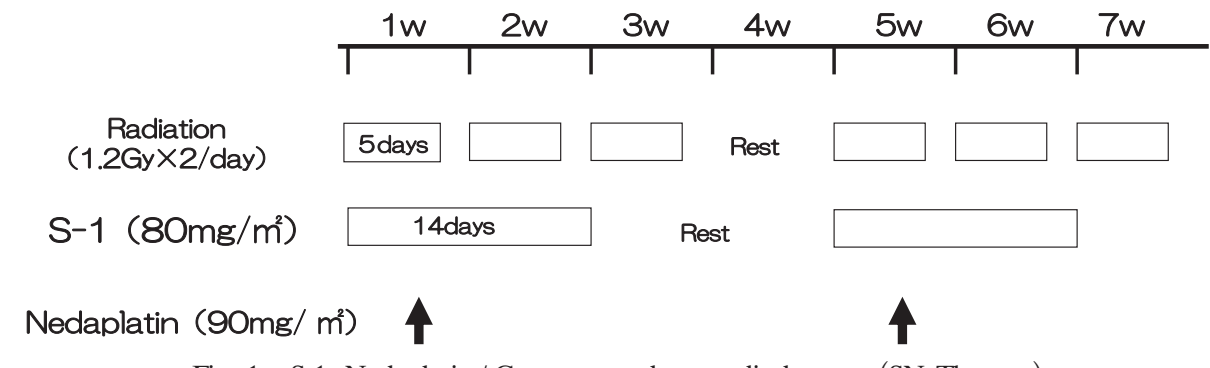

Fig. 1. S-1, Nedaplatin / Concurrent chemoradiotherapy (SN Therapy)

treatment modalities for such patients.

In this department, we provide treatment for head and neck malignancies with the intention of a permanent cure with preservation of organs and function. We report the treatment outcomes in five cases of maxillary cancer for which we utilized S-1 and nedaplatin with concurrent radiation therapy (SN therapy) from April 2005 to March 2009.

\section{Patients and Methods}

We administered SN therapy to five patients with maxillary cancer in this department from April 2005 to March 2009. With regard to histological type, all cases were identified as squamous cell carcinoma.

The research parameters were age, sex, follow-up duration, TNM Classification (UICC Classification, 2002), staging classification and treatment type.

The SN regimen used involved two courses, each consisting of a 14-day oral administration of S-1 $\left(80 \mathrm{mg} / \mathrm{m}^{2}\right)$, an intravenous infusion of Nedaplatin $\left(90 \mathrm{mg} / \mathrm{m}^{2}\right)$ on the fourth day and radiation therapy $(1.2 \mathrm{~Gy} \times 2 /$ day $)$ for three weeks. The second course was initiated after a one-week break ${ }^{1,2}$ (Fig. 1). Radiation therapy consisted of a total of 69.6 Gy. SN therapy is indicated for patients younger than 80 years old with a creatinine clearance of more than $60 \mathrm{ml} /$ minute and the absence of serious liver or cardiac disease. For patients over 75 years old the dosage is reduced to $70-80 \%$ of the normal dosage. Cisplatin, 5-FU and combination therapy with radiation are performed before SN therapy in our department. For renal function disorders cisplatin is replaced by nedaplatin.

The therapeutic protocol for maxillary cancer in this department is to administer radiation therapy after excision of the maxillary tumor in cases of T1 tumors. For T2 and T3 cases, SN therapy is provided after excision of the maxillary tumor. In T4 cases, one course of SN therapy is provided and excision of the maxillary tumor is performed two weeks later followed by the second course of SN therapy (Fig. 2). 〔All the operations involved a Denker's procedure.]

Age / Sex

The patient ages ranged from 29 to 67 years and the average age was 55.6 years. The 


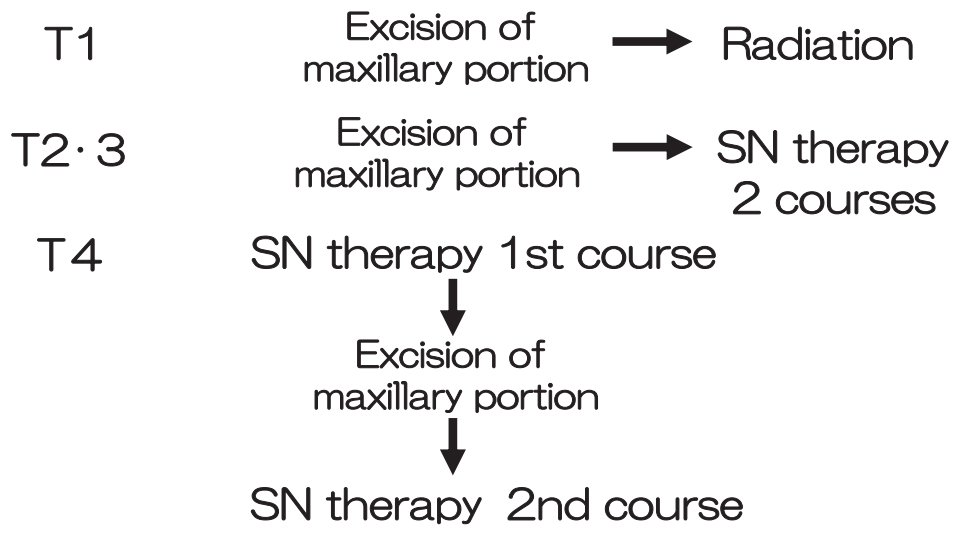

Fig. 2. Therapeutic strategy for maxillary cancer

Table 1. Details of Treatment

\begin{tabular}{|c|c|c|c|c|c|}
\hline & Age / Sex & classification & $\begin{array}{l}\text { Progress after } \\
\text { SN therapy }\end{array}$ & $\begin{array}{l}\text { Follow-up } \\
\text { (month) }\end{array}$ & Outcome \\
\hline 1 & $62 \mathrm{M}$ & $\mathrm{T} 4$ & $\begin{array}{l}\text { adjuvant chemotherapy } \\
\text { (S-1) }\end{array}$ & $31 \mathrm{~m}$ & $\begin{array}{l}\text { surviving without } \\
\text { cancer }\end{array}$ \\
\hline 2 & $29 \mathrm{M}$ & $\mathrm{T} 4$ & $\begin{array}{c}\text { Neck LN metastasis } \\
\text { Neck dissection } \\
\text { adjuvant chemotherapy } \\
\text { (UFT) }\end{array}$ & $45 \mathrm{~m}$ & $\begin{array}{l}\text { surviving without } \\
\text { cancer }\end{array}$ \\
\hline 3 & $58 \mathrm{M}$ & $\mathrm{T} 2$ & None & $33 \mathrm{~m}$ & $\begin{array}{l}\text { surviving without } \\
\text { cancer }\end{array}$ \\
\hline 4 & $60 \mathrm{M}$ & $\mathrm{T} 4$ & $\begin{array}{l}\text { Neck LN metastasis } \\
\text { Neck dissection }\end{array}$ & $37 \mathrm{~m}$ & $\begin{array}{l}\text { surviving without } \\
\text { cancer }\end{array}$ \\
\hline 5 & $62 \mathrm{M}$ & $\mathrm{T} 4$ & $\begin{array}{l}\text { Arterial injection } \\
\text { Cyber knife } \\
\text { Lung metastasis }\end{array}$ & $15 \mathrm{~m}$ & $\begin{array}{l}\text { surviving with } \\
\text { cancer }\end{array}$ \\
\hline
\end{tabular}

All cases were NO MO.

median age was 60 years of age. All patients were male.

\section{Follow-up Duration}

The follow-up duration ranged from 15 months to 45 months and the average follow-up period was 32.4 months. The median value was 33 months.

TNM Classification/Staging Classification (UICC Classification, 2002)

Four cases were classified as T4NOMO (Stage IV) and one case as T2NOMO (Stage II). 


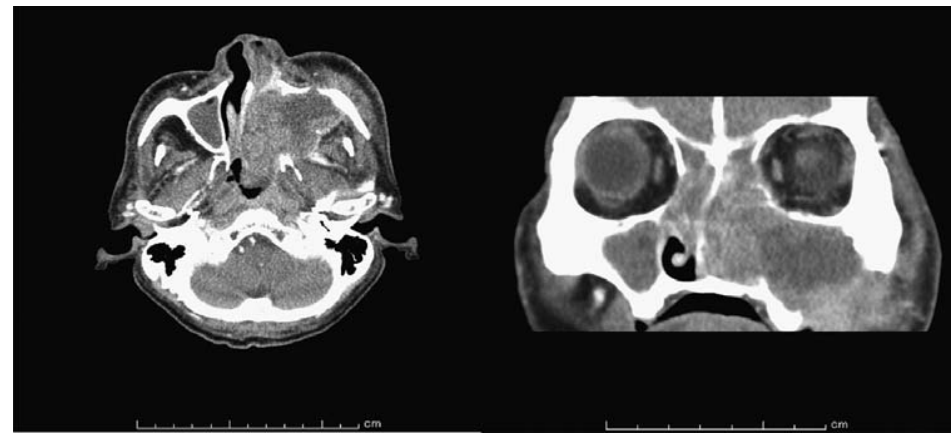

Fig. 3. Sinus CT at first examination

A tumor progressing to the oral cavity and the right nasal cavity from the left maxillary sinus.

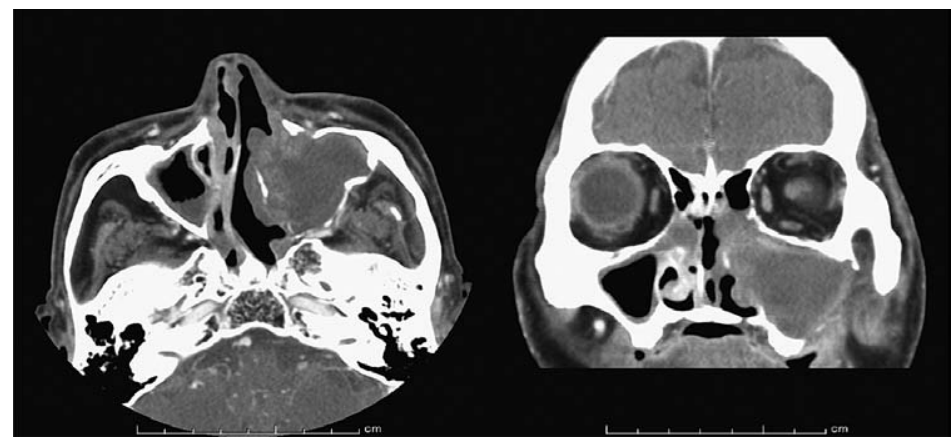

Fig. 4. Sinus CT after one course of SN therapy The tumor is reduced.

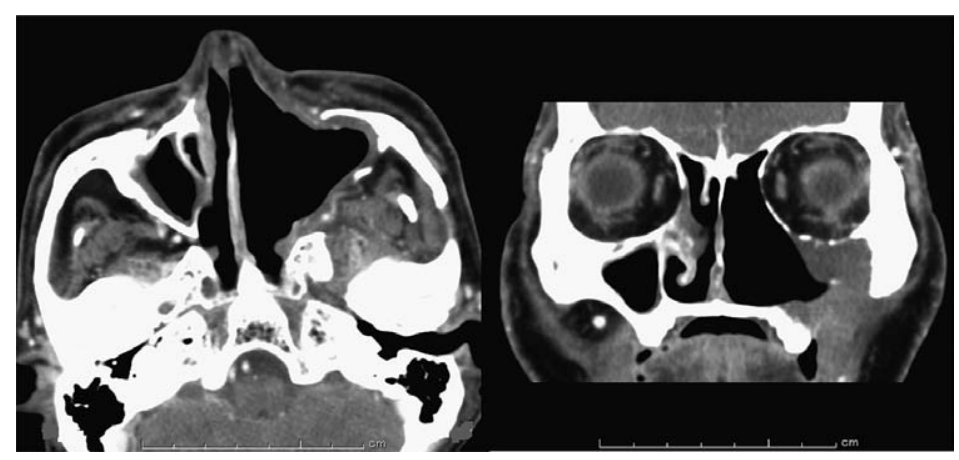

Fig. 5. Sinus CT after treatment The tumor has disappeared.

\section{Results}

Treatment Details (Table 1)

The T2 case underwent an excision of the maxillary tumor followed by SN therapy. The observation period was 33 months and the patient is presently living without cancer. (case 3) 
Four cases were classified as T4. These patients received one course of SN before undergoing an excision of the maxillary tumor. The second course of SN therapy was then administered. Three of these cases achieved a complete response (CR). In one case, the administration of S-1 as an adjunctive chemotherapy was performed for two weeks followed by a two-week break. This pattern was performed for 12 months. The observation period was 31 months and the patient is presently living without cancer. (case 1, Fig. 3, 4, 5)

In another case, UFT was administered as an adjunctive chemotherapy. After ten months a cervical lymph node metastasis appeared. This was surgically removed. The observation period was 45 months and the patient is presently living without cancer. (case 2)

Another of the T4 cases experienced a cervical lymph node metastasis after six months. This tumor was also removed surgically. The observation period was 37 months and the patient is currently living without cancer. (case 4)

The remaining case did not achieve a CR. This patient received an arterial injection of docetaxel by the Seldinger method, followed by an arterial injection of 5FU from a superficial temporal artery. A partial response (PR) was achieved. The patient then underwent CyberKnife treatment (total $3500 \mathrm{cGy}$ ), which resulted in local control, but lung metastasis subsequently occurred. The follow-up duration was 15 months and the patient is currently living with cancer. (case 5)

\section{Discussion}

Tumors of the nasal cavity and paranasal sinus can be benign or malignant and can vary histologically. According to our tumor statistics, $38.5 \%$ of these tumors are benign and $61.5 \%$ are malignant. Papillomas comprise $25.0 \%$ of cases, squamous cell carcinoma comprises $21.3 \%$, malignant lymphoma comprises $19.3 \%$, pseudotumors comprise $11.6 \%$, malignant melanoma, malignant fibrous histiocytoma, rhabdomyosarcoma and plasmacytoma each comprise 3.8\%, and hamartoma, hamangioma, small cell cancer and adenocarcinoma each comprise $1.9 \%$.

Since the report of combined therapy by Sato et $a l^{3)}$, there has been significant progress with regard to the treatment of maxillary cancer, however inter-facility differences in therapeutic strategies exist. There are several different methods for performing an en bloc resection and combined therapy. At present, the use of medical treatments such as arterial injection chemotherapy, fractional excision and concurrent chemoradiotherapy vary depending on facility protocols.

In this department, we have achieved favorable outcomes ${ }^{1)}$ using $\mathrm{SN}$ therapy for cancer of the head and neck ${ }^{2}$. In cases of maxillary cancer, we preserve the palate and eyeball with a combination of SN therapy and partial resection of the maxilla. In four of the five cases in this report, we were able to reduce the extent of surgery by administering SN therapy. Function and morphology could therefore be preserved. In the remaining case, however, an internal carotid invasion had already occurred at the time of diagnosis, and 
radical resection was impossible. Although we provided medical treatment according to departmental policy, the tumor persisted even after arterial injection chemotherapy. This patient developed a lung metastasis which we controlled locally with CyberKnife treatment. This outcome demonstrates that CyberKnife treatment is a viable treatment option for cases of recurrence and PR.

Previous studies have reported five-year survival rates for maxillary cancer patients ranging from $45.0 \%-62.5 \%$, regardless of treatment variability ${ }^{6}$. For the preservation of the palate and eyeball however, outcomes vary between facilities and a rate of maxillary preservation of about $30.0 \%$ has been reported ${ }^{3)}$. In this department, we administer SN therapy and perform partial resection of the maxilla. The palate is preserved via resection of the sidewall of the nasal cavity only. This treatment strategy maintains the QOL of patients by preserving function and morphology. However, it is difficult to make comparisons with other reports with regard to the number of cases and follow-up duration. We hope to increase the number of patients in future studies to add weight to these results.

\section{Conclusions}

We reported the outcomes of SN therapy for five cases of maxillary cancer. SN therapy allowed a reduction in the extent of surgery in order to preserve function and morphology. To assess the effectiveness of this strategy, evaluation of the survival rates in addition to the effect preserving function and morphology is required. Towards this goal, we will study an increased number of such cases in the future.

\section{References}

1) Takemura H, Iida M, Kobayashi S, Katuno M, Asano R, Kubota $Y$, Kadokura $Y$ and Sanbe T: Change of chemotherapy to stage III \& IV of laryngeal cancer and the therapeutic effect. Head Neck Cancer 31: 13491356 (2005) (in Japanese)

2) Shimane $T$, Mori $T$, Ono $T$, Monden $T$, Kobayasi S, Sanbe $T$ and Suzaki H: Treatment of cancer of the tongue in our department. Head Neck Cancer 35:5-8 (2009) (in Japanese)

3) Sato Y, Morita M, Takahashi H, Tei T, Watanabe $\mathrm{T}$ and Egawa $\mathrm{J}$ : Combined therapy for maxillary sinus carcinoma. Otol Fukuoka $15: 312-323$ (1970) (in Japanese)

4) Yamashita H, Fujii M, Ishiguro R, Tashiro M, Ohno Y, Tokumaru Y, Kanke M, Imanishi J, Tomita T, Kanzaki $\mathrm{H}$ and Inuyama $\mathrm{M}$ : Statistical analysis of maxillary sinus squamous carcinoma. J Otolaryngol Jpn 105:732740 (2002) (in Japanese)

5) Terayama Y, Yonemoto M, Osafune H and Oda J : Clinical study of maxillary sinus carcinoma. Otolaryngol Head Neck Surg 73:683-687 (2001) (in Japanese)

6) Tateda M, Yoshida F, Sajyo S, Shiga K and Yokoyama J : A clinical study of malignant maxillary sinus tumors. Head Neck Cancer 30:8-12 (2004) (in Japanese) 